\title{
Abordaje multidisciplinario de un paciente con Agenesia del cuerpo calloso.
}

Multidisciplinary approach to a patient with agenesis of the corpus callosum.

\author{
Cirit Matheus ${ }^{1}$, Elisama Beltran ${ }^{1}$, Francis Araque ${ }^{1}$
}

\section{RESUMEN}

Se describe la evolución clínica de cuatro años del caso de un paciente atendido de urgencia, por primera vez, a los 17 días de nacido, con manifestaciones de crisis convulsiva y desnutrición. Durante su hospitalización, exámenes de neuroimágenes condujeron al diagnóstico de Agenesia del cuerpo calloso (ACC), malformación que puede ocurrir de forma aislada o en asociación con otras alteraciones sistémicas o del sistema nervioso central. ACC es considerada una de las malformaciones cerebrales más frecuentes, con una prevalencia estimada en 1 de 4000 nacidos vivos. Se revisan los aportes teóricos y clínicos sobre la enfermedad para concluir que la importancia del caso radica en que las estrategias interdisciplinarias utilizadas en el tratamiento han generado cambios significativos en la evolución clínica de ACC. Asimismo, el compromiso y participación de los padres en la implementación de cuidados paliativos, reviste significativa importancia.

PALABRAS CLAVE: cuerpo calloso, crisis convulsiva, adhesión al tratamiento, cuidados paliativos.

\section{SUMMARY}

The four-year clinical evolution of a patient seen for the first time at the age of 17 days, when he was admitted to the emergency room due to seizures and malnutrition, is described. During this hospitalization, neuroimaging tests led to the diagnosis of Agenesis of the corpus callosum (ACC), a malformation that can occur in isolation or in association with other systemic or central nervous system conditions. ACC is considered one of the most frequent brain malformations, with an estimated prevalence of 1 in 4000 live births. The main theoretical and clinical contributions about the disease are reviewed. It is concluded that the importance of the case lies in the fact that the interdisciplinary strategies used in the treatment of ACC patients have generated significant changes in their clinical evolution. As well, the commitment and participation of the parents in the application of palliative care is of significant importance.

KEY WORDS: corpus callosum, seizure, adherence to treatment, palliative care.

1 Universidad Metropolitana. Barranquilla, Colombia. 


\section{INTRODUCCIÓN}

La agenesia del cuerpo calloso (ACC), es la falta de formación de la región cerebral llamada cuerpo calloso, producto de una alteración en el desarrollo embrionario a partir de la semana 20 que ocasiona la falta parcial o total de este importante haz de fibras interhemisféricas cerebrales. Esta condición clínica fue descrita por primera vez por Albert Schinzel en 1979 (1), y en la actualidad existen estudios exhaustivos de su etiopatogenia, sus comorbilidades y su desarrollo. Un estudio llevado durante el período de 29 años de la prevalencia general de ACC fue de 2,56 por 10000 nacimientos, lo cual no la hace una patología muy frecuente (2), a menudo se asocia a otras malformaciones congénitas, puede ocurrir de forma aislada o en compañía de otras alteraciones del sistema nervioso central o sistémico como por ejemplo existe una proporción de agenesia / hipoplasia 5,7 (217/38), y las malformaciones asociadas con mayor frecuencia son las del sistema musculoesquelético y las trisomías son las anomalías cromosómicas más frecuentes, en particular la trisomía18 y 13 (respectivamente 9/32 y $4 / 32$ casos), los estudios muestran mayor riesgo para los hombres (3).

Desde el punto de vista etiopatogénico se le atribuye a múltiples factores de orden genético, incluyen errores cromosómicos, infecciones prenatales y otros factores relacionados con el entorno prenatal, tales como el consumo de alcohol en la madre (4) se ha identificado, a través de la secuenciación del exoma completo (WES), dos mutaciones genéticas en el cromosoma X: una es una mutación sin sentido hemizigótica c.3746T > C (p. L1249P) en la subunidad del complejo mediador génico 12 (MED12); la otra es una mutación sin sentido.

Las manifestaciones clínicas corresponden a diferentes variantes de malformación del cuerpo calloso, entre ellas existen ACC total, ausencia del CC, ACC parcial, la hipoplasia donde la dirección de crecimiento del cuerpo calloso está presente, pero los nervios no se desarrollan como para formar una estructura y la heterocigota c. $128+5 \mathrm{G}>\mathrm{C}$ en el gen ephrin B1 (EFNB1) (5); otros factores asociados son causas genéticas, infecciosas, vasculares o tóxicas , y también la disgenesias del cuerpo calloso (6).

Al mismo tiempo la ACC, puede estar presentarse con algunos síndromes epilépticos como son: Síndrome de West, Lennox Gastaut, síndrome de Otahara, (7) también suele caracterizarse por retardo general en el desarrollo psicomotor, relacionado con la coordinación, equilibrio, hipotonía; así como alteraciones cognitivas relevantes, por consiguiente deterioro del razonamiento abstracto, dificultad en la resolución de problemas, retraso en el lenguaje, dificultad de expresarse con coherencia, dificultades en el lenguaje comprensivo, son niños que presentan desde un coeficiente intelectual menor de lo esperado hasta un retardo mental severo (8).

Los resultados de estudios cognitivos y neurológicos en pacientes con ACC suelen ser normales o con problemas de comportamiento leves en algunos casos, sin embargo, el pronóstico es peor en casos con malformaciones cerebrales asociadas (9). Estudios recientes de seguimiento a largo plazo muestran un resultado normal del desarrollo neurológico o discapacidades leves en el $65-88 \%$ de los ACC (10).

También se ha descrito que algunos pacientes presentan cognición social deficiente ocasionando problemas para integrar información de múltiples fuentes (verbal y visual) y usar señales paralingüísticas para la emoción y comprender el habla no literal (11).

Desde el punto de vista clínico pueden presentar crisis convulsivas frecuentes (tipo motora, clónica), macrocefalia, hipertelorismo, parálisis cerebral, déficit neurológico focal, síndrome de desconexión (12). Con respecto al diagnóstico, una de las complicaciones es que el trastorno solo se puede diagnosticar firmemente a través de la neurorradiología (13).

Debido a esto el abordaje terapéutico no se encuentra estandarizado en tanto que depende de las características de cada paciente y las comorbilidades, los compromisos o déficits neurológicos, cognitivos, sociales, de comportamiento, entre otros. Si existen crisis convulsivas es importante su control, en razón que estas pueden ocasionar un mayor deterioro de las funciones cerebrales es por esta complejidad del cuadro que se plantea el requerimiento de un equipo interdisciplinar para el tratamiento de estos casos.

De acuerdo a los antecedentes teórico-prácticos la intervención se enfoca en las directrices dadas en la tabla 1; el seguimiento integral en el caso clínico ACC requiere ambientes estructurados y con proyectos claramente definidos, buscando la potenciación máxima de las posibilidades físicas e intelectuales del niño mediante la estimulación regulada y continuada, llevada a cabo en todas las áreas del desarrollo, con 
Tabla 1. Evolución actual del paciente

\begin{tabular}{ll}
\hline Motor grueso & Se coloca de pie \\
& Se sienta \\
Gatea & Da pasos agarrado de la baranda de la cuna \\
& Usa los dedos y manos para exploración \\
Agarra objetos incluyendo una cuchara, aunque no la maneja con \\
destreza \\
\\
Rotor fino & Realiza interacción con el medio \\
& Pide algunos alimentos por su nombre (tete, agua) \\
Área social & Comprende el lenguaje y se hace entender con algunas palabras \\
& y señas. \\
Lenguaje & Expresiones faciales y manifestaciones emocionales \\
\hline
\end{tabular}

Fuente: Elaboración propia de las autoras (2021)

la participación de los padres, mostrándoles la forma correcta de realizar los ejercicios de tal manera que formen parte activa en el tratamiento de su hijo, de allí que el manejo de apoyo específico de los síntomas, la mejora del crecimiento y el desarrollo y las intervenciones de apoyo de los padres son clave para manejar a los niños con agenesia del cuerpo calloso (14).

El presente caso clínico ha sido tratado con abordaje multidisciplinar en la FHUM (Fundación Hospital Universitario Metropolitano de Barranquilla, Colombia), cuyo objetivo es fundamentar la evolución del caso, con una trazabilidad de 4 años, describiendo las estrategias empleadas en la intervención multidisciplinar realizadas, en este sentido se busca fortalecer la efectividad del proceso de intervención, involucrando un equipo interdisciplinar de profesionales que proporcionaron cambios favorables en la salud y la calidad de vida del paciente y su familia.

\section{Presentación del caso}

Se trata de un menor de 4 años, con antecedentes de múltiples hospitalizaciones desde sus primeros días de nacido, por crisis epilépticas, y problemas para tragar, según antecedentes perinatales, la madre niega patologías durante el embarazo e ingesta de medicamentos, u otro tipo de sustancias psicoactivas; asevera control prenatal durante el tiempo de gestación de forma puntual. Menor producto del tercer embarazo a término 37,5 semanas, por vía vaginal, con un peso al nacer de 2300 gramos, y medidas de $35 \mathrm{~cm}$, alimentado a través de leche materna y leche de formula con mezcla de cereal. Al realizar la exploración respectiva se observa retraso en el desarrollo psicomotor: sostén cefálico a los 5 meses y dificultades para la sedestación, síndrome febril agudo y microcefalia. Tratado farmacológicamente con fenobarbital $25 \mathrm{mg}$ 1 diaria vía oral. La conducta sugerida y atención multidisciplinar se encuentran expuestas en la tabla 2 y tabla 3 de acuerdo a la evolución del cuadro clínico.

\section{Metodología}

El seguimiento del caso se desarrolló a través de la recolección de información documental con fuentes primarias como lo es la historia clínica, combinado con observación directa, entrevistas con los familiares y algunos profesionales de atención domiciliaria, consulta externa. Lo que permitió tener acceso a la información relacionada con los diferentes procedimientos, pruebas de laboratorios e imágenes diagnósticas. Se realizó un análisis de tipo longitudinal, en tanto se hizo seguimiento al caso durante cuatro años, se fueron contrastando los hallazgos clínicos, con los diferentes signos y síntomas, y el desarrollo del niño en el tiempo observado. Todo ello bajo la modalidad de estudio de caso.

El compromiso de los padres en las asistencias médicas periódicas ha sido el esperado, sin embargo, tropiezan con el obstáculo de solo contar con un régimen subsidiado, no contributivo, lo que ha traído como consecuencia que los tratamientos se trunquen, los trasladen de empresa prestadora de servicios de salud, entre otros inconvenientes. Causando muchas veces disrupciones en la en las intervenciones clínicas necesarias. No obstante, lo diferencial de este caso, 
Abordaje multidisciplinario de un paciente con Agenesia del cuerpo calloso.

según lo expresado anteriormente, está determinado porque los padres se encuentran apropiados en adoptar, mantener y aplicar las sugerencias y formación recibidas por cada profesional, reflejándose en el estado general actual del paciente.
Actualmente el paciente cuenta con 4 años, su evolución clínica muestra evolución satisfactoria, en lo que respectan los estados convulsivos, se han presentado de manera episódica a medida que va creciendo, por lo tanto, debe ajustarse la cantidad de

Tabla 2. Seguimiento multidisciplinar en el caso clínico ACC.

\begin{tabular}{|c|c|c|c|}
\hline $\begin{array}{l}\text { Profesionales de salud } \\
\text { que intervienen }\end{array}$ & Conducta a seguir & Objetivo De Cada Profesional & Efectos en el caso ACC \\
\hline PEDIATRA & $\begin{array}{c}\text { Exploración } \\
\text { neurofisiológica: } \\
\text { Potenciales evocados } \\
\text { auditivos, visuales, } \\
\text { cognitivos y } \\
\text { somatosensoriales } \\
\text { ordena intervención } \\
\text { integral }\end{array}$ & $\begin{array}{l}\text { Seguimiento del caso: desarrollo } \\
\text { evolutivo del niño. } \\
\text { Medicación respectiva y } \\
\text { adherencia }\end{array}$ & $\begin{array}{l}\text { Claridad en la evolución clínica del menor } \\
\text { en relación al desarrollo evolutivo }\end{array}$ \\
\hline \multirow[t]{2}{*}{ NEURÓLOGO } & $\begin{array}{l}\text { Encefalografía digital } \\
\text { Resonancia Magnética }\end{array}$ & $\begin{array}{l}\text { Confirmar malformación } \\
\text { congénita del encéfalo }\end{array}$ & $\begin{array}{l}\text { Facie con trigonocefalia } \\
\text { Parpados edematosos } \\
\text { Epicanto } \\
\text { Fisuras oblicuas } \\
\text { Se encuentra constituida por un ritmo alfa } \\
\text { de } 8-10 \text { hz de frecuencia y una amplitud, } \\
\text { hallazgo frecuente en ACC }\end{array}$ \\
\hline & $\begin{array}{c}\text { Fenobarbital } 25 \mathrm{mg} 1 \\
\text { diaria vía oral } \\
\text { Ácido Valproico de } \mathrm{mg} / \mathrm{kg} / \\
\text { día * por tres días } \\
* \text { (indica que para } 1 \mathrm{~kg} \mathrm{de} \\
\text { peso se administran } 10 \mathrm{mg} \text { ) }\end{array}$ & $\begin{array}{l}\text { Reducir crisis epilépticas, } \\
\text { Psicoeducación con los padres } \\
\text { Lograr adherencia optimizada al } \\
\text { tratamiento farmacológico. }\end{array}$ & $\begin{array}{l}\text { Control de las crisis epilépticas } \\
\text { Logro a la adherencia optimizada }\end{array}$ \\
\hline GENETISTA & $\begin{array}{l}\text { Análisis cromosómico } \\
\text { Cariotipo RT }\end{array}$ & $\begin{array}{l}\text { Confirmar malformación } \\
\text { congénita del encéfalo }\end{array}$ & $\begin{array}{l}\text { Darle continuidad al tratamiento } \\
\text { multidisciplinar }\end{array}$ \\
\hline GASTROENTERÓLOGO & $\begin{array}{l}\text { Estudios de deglución: } \\
\text { Faringografia y } \\
\text { esofagograma }\end{array}$ & $\begin{array}{l}\text { Determinar las causas del } \\
\text { Reflujo gastroesofágico grado IV }\end{array}$ & $\begin{array}{l}\text { El esófago muestra calibre contornos y } \\
\text { pliegues mucosos normales, sin imágenes } \\
\text { de exceso ni defectos de llenado, no hubo } \\
\text { extravasación del medio de contraste. } \\
\text { Se observó reflujo gastroesofágico hasta la } \\
\text { porción cervical del esófago. }\end{array}$ \\
\hline FISIOTERAPIA & $\begin{array}{l}\text { Terapia física en el } \\
\text { manejo de: } \\
\text { Fuerza muscular } \\
\text { Valoración de las } \\
\text { amplitudes articulares } \\
\text { Tono pasivo de los } \\
\text { músculos, } \\
\text { ángulo de abductores, } \\
\text { ángulo de dorsiflexion de } \\
\text { tobillos. } \\
\text { Aplicar técnica de } \\
\text { Cinesiterapia }\end{array}$ & $\begin{array}{l}\text { Rehabilitación para el Retraso } \\
\text { psicomotor grave enfocado a: } \\
\text { Hipertonía en ambas } \\
\text { extremidades- movimientos } \\
\text { incontrolados } \\
\text { Pie en valgo } \\
\text { Aplicar estimulación global e } \\
\text { integración sensomotriz } \\
\text { Entrenamiento de los padres }\end{array}$ & $\begin{array}{l}\text { Mejoría en la actitud postural del niño, } \\
\text { Control en cabeza- tronco y brazos. } \\
\text { Respuesta a la estimulaciones táctil, visual } \\
\text { y vestibular } \\
\text { Se mantiene sentado mayor parte del } \\
\text { tiempo } \\
\text { Menor espasticidad de miembros inferiores } \\
\text { Rutinizar las tareas repetitivas y } \\
\text { convertirlas en automáticas. } \\
\text { Formación a los padres en la forma } \\
\text { correcta de realizar los ejercicios. }\end{array}$ \\
\hline TERAPIA FÍSICA & $\begin{array}{c}\text { Terapia física a nivel } \\
\text { neuromusculoesquelético y } \\
\text { motricidad fina } \\
\text { Lateralización }\end{array}$ & $\begin{array}{l}\text { Estimular el sistema } \\
\text { neuromusculoesquelético } \\
\text { Lograr segmentación y } \\
\text { automatización. } \\
\text { Mejorar el conocimiento y } \\
\text { reconocimiento corporal } \\
\text { Entrenamiento de los padres } \\
\text { Lateralización indiferenciada }\end{array}$ & $\begin{array}{l}\text { Capaz de girarse en la cama solo } \\
\text { Girarse la cabeza en busca del sonido o } \\
\text { estimulo auditivo. } \\
\text { Logra sostener objetos con una sola mano, } \\
\text { ambas y alternos. } \\
\text { Mejoría en la exploración del tacto. } \\
\text { Separación de los dedos de las manos } \\
\text { Mayor elección del brazo derecho para } \\
\text { ejecutar tareas. }\end{array}$ \\
\hline
\end{tabular}


Tabla 2 (cont). Seguimiento multidisciplinar en el caso clínico ACC.

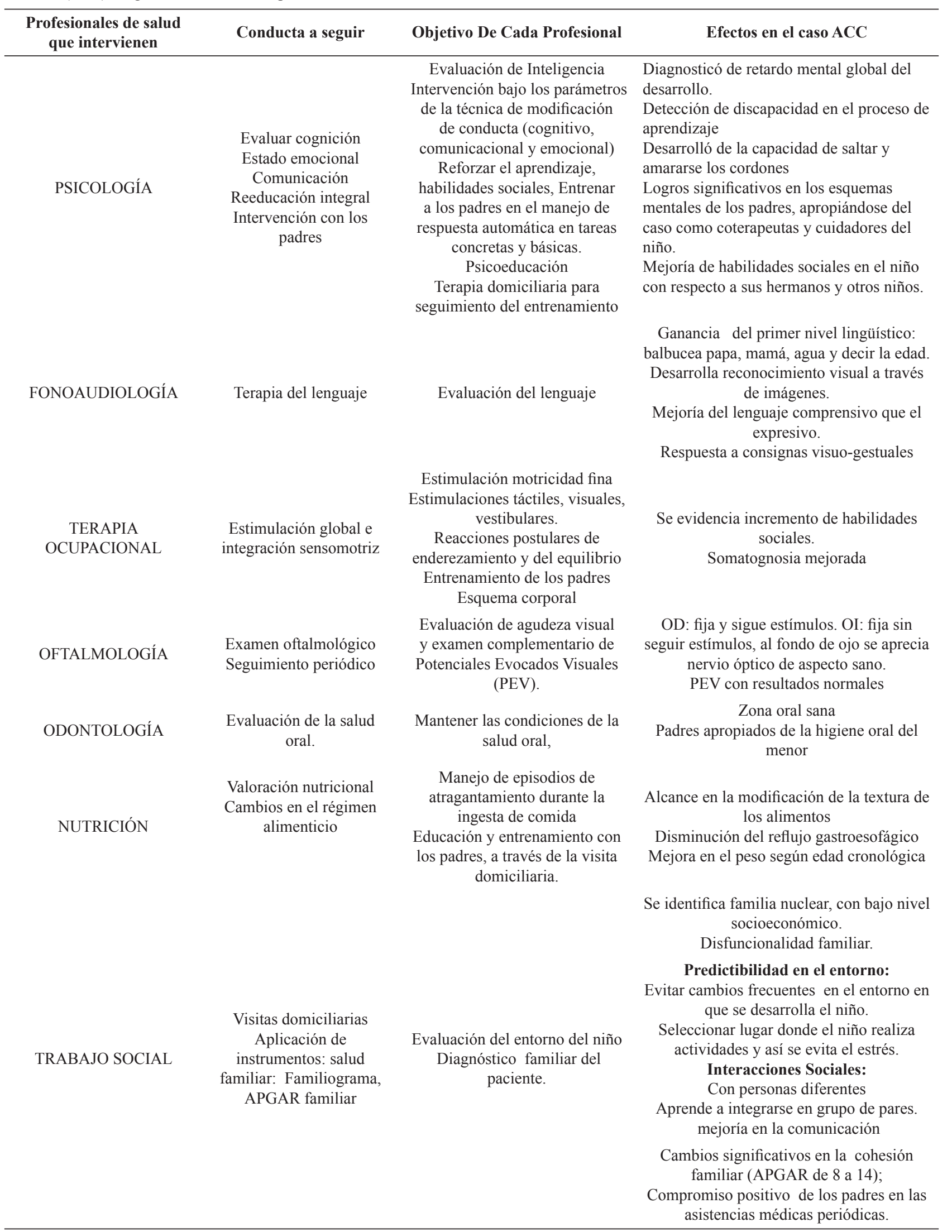


Abordaje multidisciplinario de un paciente con Agenesia del cuerpo calloso.

Tabla 3. Trazabilidad del caso clínico: ACC, desde los 17 días de nacido - 4 años de edad por equipo multidisciplinar.

\begin{tabular}{|c|c|c|c|c|}
\hline $\begin{array}{c}\text { Edad de } \\
\text { intervención }\end{array}$ & $\begin{array}{l}\text { Motivo de la } \\
\text { intervención }\end{array}$ & Intervención & Diagnóstico & Tratamiento \\
\hline $\begin{array}{l}17 \text { días de } \\
\text { nacido }\end{array}$ & $\begin{array}{l}\text { Convulsiones y } \\
\text { dificultad para tragar }\end{array}$ & $\begin{array}{l}\text { Pediatría y } \\
\text { neurología }\end{array}$ & $\begin{array}{l}\text { Agenesia del cuerpo calloso } \\
\text { Retardo e la mielinización }\end{array}$ & $\begin{array}{l}\text { Seguimiento del caso: desarrollo } \\
\text { evolutivo del niño. }\end{array}$ \\
\hline 1 mes 11 días & $\begin{array}{l}\text { Pie plano laxo infantil } \\
\text { funcional }\end{array}$ & Fisioterapia & Retardo en el desarrollo & $\begin{array}{c}\text { Terapia física en el manejo de: } \\
\text { Fuerza muscular } \\
\text { Valoración de las amplitudes } \\
\text { articulares Tono pasivo de los } \\
\text { músculos } \\
\text { Ángulo de abductores, ángulo de } \\
\text { dorsiflexión de tobillos. } \\
\text { Aplicar técnica de Cinesiterapia } \\
30 \text { sesiones una por día }\end{array}$ \\
\hline \multirow[t]{2}{*}{2 meses } & $\begin{array}{l}\text { Convulsiones } \\
\text { Movimientos tónico } \\
\text { generalizado } \\
\text { Perdida del tono } \\
\text { muscular }\end{array}$ & Hospitalización & $\begin{array}{c}\text { Crisis convulsiva } \\
\text { Agenesia del cuerpo calloso } \\
\text { Síndrome dismórfico genético } \\
\text { Hipospadia }\end{array}$ & $\begin{array}{l}\text { Hospitalización en UCI neonatal } \\
\text { Fenobarbital ampollas } 25 \mathrm{mg} / \mathrm{kg} \text { x día }\end{array}$ \\
\hline & $\begin{array}{l}\text { Convulsiones y } \\
\text { cuadro febril }\end{array}$ & Pediatría & $\begin{array}{c}\text { Hipospadia Pie en valgo } \\
\text { Facie con Trigonocefalia Orejas } \\
\text { bajas }\end{array}$ & $\begin{array}{l}\text { Seguimiento del caso: desarrollo } \\
\text { evolutivo del niño. }\end{array}$ \\
\hline & Brote en Pliegues & Dermatología & Dermatitis Atópica & Tratamiento tópico en región afectada \\
\hline 5 meses & $\begin{array}{c}\text { Confirmar } \\
\text { malformación } \\
\text { congénita del encéfalo }\end{array}$ & Genetista & $\begin{array}{l}\text { Malformación congénita } \\
\text { múltiples Trigonocefalia } \\
\text { Hipospadia }\end{array}$ & $\begin{array}{l}\text { Análisis cromosómico } \\
\text { Cariotipo RT }\end{array}$ \\
\hline $\begin{array}{l}6 \text { meses de } \\
\text { vida }\end{array}$ & Convulsiones & $\begin{array}{c}\text { Hospitalización } \\
\text { Equipo de pediatría } \\
\text { de UCI }\end{array}$ & $\begin{array}{l}\text { Dificultad en sostén cefálico } \\
\text { Reflejos oculocefálicos }\end{array}$ & $\begin{array}{l}\text { Hospitalización en UCI neonatal } \\
\text { Fenobarbital ampollas } 25 \mathrm{mg} / \mathrm{kg} \text { x día }\end{array}$ \\
\hline \multirow[t]{2}{*}{8 meses } & $\begin{array}{l}\text { Movimientos tónico } \\
\text { generalizado } \\
\text { Perdida del } \\
\text { conocimiento }\end{array}$ & Hospitalización & $\begin{array}{c}\text { Crisis convulsiva } \\
\text { ACC } \\
\text { Deformidad en valgo pie derecho } \\
\text { Desnutrición }\end{array}$ & $\begin{array}{l}\text { Orden de exámenes de laboratorio } \\
\text { Remisión a psicología y Nutrición }\end{array}$ \\
\hline & & Oftalmología & Visión subnormal de ambos ojos & Potenciales visuales evocados \\
\hline 11 meses & $\begin{array}{l}\text { Valoración por } \\
\text { antecedentes clínicos }\end{array}$ & Genetista & $\begin{array}{c}\text { Malformación congénita del } \\
\text { encéfalo } \\
\text { Trigonocefalia } \\
\text { Parpados edmatosis } \\
\text { Fisuras Oblicuas } \\
\text { Pliegue del Epicanto } \\
\text { Filtrum marcado }\end{array}$ & $\begin{array}{c}\text { Análisis cromosómico } \\
\text { Cariotipo RT } \\
\text { Microarreglos: MICHOCHIP } \\
\text { CYTOSCAN HD AFFYMETRIX }\end{array}$ \\
\hline 12 meses & $\begin{array}{l}\text { Evaluar cognición } \\
\text { Estado emocional } \\
\text { Reeducación integral } \\
\text { Evaluar comunicación }\end{array}$ & Psicología & $\begin{array}{c}\text { Retardo mental global del } \\
\text { desarrollo } \\
\text { Discapacidad en el aprendizaje }\end{array}$ & $\begin{array}{c}\text { Terapia Multidisciplinaria y } \\
\text { domiciliaria (acompañamiento a los } \\
\text { padres) } \\
\text { Seguimiento terapéutico con los } \\
\text { padres }\end{array}$ \\
\hline 13 meses & $\begin{array}{l}\text { Convulsiones } \\
\text { Movimientos tónico } \\
\text { generalizado Perdida } \\
\text { del conocimiento }\end{array}$ & Hospitalización & $\begin{array}{l}\text { Crisis convulsiva } \\
\text { ACC } \\
\text { Microcefalia }\end{array}$ & $\begin{array}{c}\text { Orden de exámenes de laboratorio } \\
\text { Rayos X de tórax }\end{array}$ \\
\hline 18 meses & Convulsiones & $\begin{array}{l}\text { Hospitalización } \\
\text { Interconsulta con } \\
\text { Neurología }\end{array}$ & $\begin{array}{l}\text { Crisis convulsiva } \\
\text { ACC } \\
\text { Microcefalia }\end{array}$ & $\begin{array}{c}\text { Ácido Valproico } 1.5(22.5 \mathrm{Kg}) \\
\text { Potenciales evocados } \\
\text { Equipo multidisciplinar (Fisioterapia, } \\
\text { Fisiatra, } \\
\text { Psicología, Terapía Ocupacional, } \\
\text { Fonoaudiología y Terapia } \\
\text { Domiciliaria) }\end{array}$ \\
\hline
\end{tabular}


Tabla 3 (cont). Trazabilidad del caso clínico: ACC, desde los 17 días de nacido - 4 años de edad por equipo multidisciplinar.

\begin{tabular}{|c|c|c|c|c|}
\hline $\begin{array}{c}\text { Edad de } \\
\text { intervención }\end{array}$ & $\begin{array}{l}\text { Motivo de la } \\
\text { intervención }\end{array}$ & Intervención & Diagnóstico & Tratamiento \\
\hline \multirow{2}{*}{$\begin{array}{l}1 \text { año } \\
8 \text { meses }\end{array}$} & $\begin{array}{l}\text { No responde a } \\
\text { estímulos visuales }\end{array}$ & \multirow[t]{2}{*}{ Fisiatría } & $\begin{array}{l}\text { Respuesta evocada para P-100 } \\
\text { latencia y amplitud normal }\end{array}$ & $\begin{array}{c}\text { Potenciales evocados visuales } \\
\text { Potenciales evocados auditivos } \\
\text { umbrales } \\
\text { Seguimiento del desarrollo evolutivo } \\
\text { del niño. }\end{array}$ \\
\hline & $\begin{array}{l}\text { No responde a } \\
\text { estímulos auditivos }\end{array}$ & & $\begin{array}{c}\text { Onda V intensidad 20dBnHL OD } \\
\text { 40dBnHL OI } \\
\text { Pérdida de audición oído } \\
\text { izquierdo sin reproducibilidad }\end{array}$ & $\begin{array}{l}\text { Impedanciometria } \\
\text { PEAee }\end{array}$ \\
\hline $\begin{array}{l}1 \text { año } 11 \\
\text { meses }\end{array}$ & $\begin{array}{l}\text { Vómito después de las } \\
\text { comidas }\end{array}$ & Gastroenterólogo & Reflujo gastroesofágico grado IV & $\begin{array}{c}\text { Faringografia } \\
\text { Esofagograma } \\
\text { Remisión a Nutrición }\end{array}$ \\
\hline \multirow[t]{2}{*}{2 años } & \multirow[t]{2}{*}{$\begin{array}{l}\text { Seguimiento del caso } \\
\text { clínico }\end{array}$} & Neurología & $\begin{array}{l}\text { Ritmo alfa de } 8-10 \mathrm{HZ} \text { Fcia } \\
80 \mathrm{MV} \text { amplitud } \\
\text { Morfología irregular y asimétrica } \\
\text { Hondas agudas generalizadas }\end{array}$ & $\begin{array}{l}\text { Seguimiento por Neurología } \\
\text { Ácido Valproico } 1.5(22.5 \mathrm{Kg}) \\
\text { Electroencefalograma }\end{array}$ \\
\hline & & Pediatría & $\begin{array}{l}\text { Retraso global del desarrollo } \\
\text { psicomotor }\end{array}$ & $\begin{array}{l}\text { Seguimiento multidisciplinar } \\
\text { Evaluación en } 6 \text { meses }\end{array}$ \\
\hline $\begin{array}{l}2 \text { años } 6 \\
\text { meses }\end{array}$ & $\begin{array}{l}\text { Consulta por el equipo } \\
\text { multidisciplinar }\end{array}$ & $\begin{array}{l}\text { Negligencia del } \\
\text { servicio de salud }\end{array}$ & Alteración clínica por ACC & Ácido Valproico 1.5mg (15 Kg) \\
\hline $\begin{array}{l}2 \text { años } 8 \\
\text { meses }\end{array}$ & $\begin{array}{l}\text { Cuadro convulsivo } \\
\text { con una hora de } \\
\text { evolución }\end{array}$ & $\begin{array}{l}\text { Urgencia - } \\
\text { Hospitalización } \\
\text { por Pediatría y } \\
\text { Neurología }\end{array}$ & $\begin{array}{c}\text { ACC } \\
\text { Status convulsivo } \\
\text { Sin medicación } \\
\text { Sin terapia hacen } 60 \text { días por } \\
\text { profesionales } \\
\text { Terapia en casa por parte de los } \\
\text { padres }\end{array}$ & $\begin{array}{l}\text { Ácido Valproico } 2.0 \mathrm{mg}(17.5 \mathrm{Kg}) \\
\text { Seguimiento multidisciplinar }\end{array}$ \\
\hline $\begin{array}{l}3 \text { años } 8 \\
\text { meses }\end{array}$ & $\begin{array}{l}\text { Cuadro convulsivo } \\
\text { con dos episodios } \\
\text { seguidos }\end{array}$ & $\begin{array}{l}\text { Urgencia - } \\
\text { Hospitalización } \\
\text { por Pediatría y } \\
\text { Neurología }\end{array}$ & $\begin{array}{c}\text { ACC } \\
\text { Status convulsivo }\end{array}$ & $\begin{array}{l}\text { Ácido Valproico } 2.5 \mathrm{mg}(20 \mathrm{Kg}) \\
\text { Seguimiento multidisciplinar }\end{array}$ \\
\hline 4 años & $\begin{array}{l}\text { Cuadro convulsivo } \\
\text { durante pandemia } \\
\text { COVID } 19\end{array}$ & $\begin{array}{l}\text { Urgencia (atención } \\
\text { por pediatría y } \\
\text { consulta telefónica } \\
\text { neurología) }\end{array}$ & $\begin{array}{c}\text { ACC } \\
\text { Status convulsivo }\end{array}$ & $\begin{array}{l}\text { Cambio de medicación a } \\
\text { Levetiracetam } 3.0 \mathrm{mg} .\end{array}$ \\
\hline
\end{tabular}

Fuente: Elaboración propia. Historia clínica del paciente (2021).

la medicación cada determinado tiempo, en el mes de mayo de 2020 le fue cambiada la medicación.

De la misma forma, trabajo social, identificó disfuncionalidad familiar y aspectos que adolece la familia por el mismo sistema socioeconómico en que se desarrolló el seguimiento del caso. Se trabajó con Predictibilidad en el entorno, se trata de evitar cambios frecuentes en el entorno en que se desarrolla el niño, siendo reiterativo en el lugar donde el niño realiza actividades y así manejo del estrés.

Se lograron interacciones sociales con personas diferentes, para la integración del niño a grupo de pares, facilitando la comunicación en él, desde esta perspectiva de la comunicación a través de
Fonoaudiología, se alcanzó, ganancia del primer nivel lingüístico: balbucea papa, mamá, agua y su edad, desarrollando reconocimiento visual a través de imágenes, lo que permitió el alcance de un mejor lenguaje comprensivo y expresivo con apropiada respuesta a consignas visuo-gestuales.

En cuanto al equipo de profesionales no médicos, a través de Fisioterapia, se ganó, mejoría en la actitud postural del niño, control en cabeza- tronco y brazos, con respuesta positiva a la estimulaciones táctil, visual y vestibular, manteniéndose sentado la mayor parte del tiempo, lo que ha sido favorecido por la menor espasticidad de miembros inferiores, recomendándose tareas repetitivas automatizarlas y formación a los padres en la forma correcta de realizar los ejercicios. 
Conjunto a Fisioterapia articulada con el área de Terapia Física se obtuvo cambios específicos y fundamentales en cuanto girarse en la cama sin ayuda, respuesta automática a los estímulos visuales y auditivos, sostener objetos con una sola mano, ambos y alternos, con exploración del tacto en forma automatizada, separación de los dedos de las manos.

Con respecto a su evolución psicomotriz, el niño se sienta, gatea, agarra objetos, come solo, lo que indica que ha superado por completo los problemas de deglución. Aunque el niño presenta un evidente déficit en sus funciones motoras y cognitivas con respecto a su edad, no presenta recidivas ni perdida de funciones o aspectos del desarrollo alcanzados, por el contrario, su curva de aprendizaje y desarrollo progresa paulatinamente en respuesta al tratamiento.

\section{DISCUSIÓN}

La agenesia del cuerpo calloso (ACC) es un defecto cerebral congénito raro que produce una amplia variedad de deficiencias cognitivas y motoras (15), cada niño diagnosticado, es un caso distinto, ya que varía la alteración producida en esta estructura, a razón de esto, la intervención y tratamientos de rehabilitación que reciba cada niño, deben ser individualizada y periódica, lo que garantizará resultados alentadores tanto para los profesionales multidisciplinares como para sus familias.

Es bien sabido, que ciertos casos presentados acentúan su tratamiento a la parte medica Neurología y pediatría, controlando los status convulsivo, sin embargo, lo preciso es una intervención multidisciplinar oportuna, ellos a su vez, pueden prevenir ciertos síntomas de forma tal que se traten a tiempo o bien sea preparen al padre de familia al manejo de sus hijos y que estos estén avisados que el síntoma puede aparecer en cualquier momento, adoptando las medidas correctivas para el caso sin entrar en pánico (16).

En relación a la problemática expuesta, en el diagnóstico de ACC, del caso en estudio, los síntomas iniciales se manifiestan con episodios convulsivos y dificultad para tragar a los 17 días de nacido, por ello, a través de múltiples estudios y pruebas de imagenología se logra confirmar tempranamente el diagnóstico. No obstante, es importante destacar que otros estudios demuestran que los niños con síndrome de Aicardi tienen un inicio más temprano de las convulsiones, un peor resultado del desarrollo y áreas más grandes de anormalidades cerebrales en la neuroimagen en comparación con la agenesia no sindrómica de los pacientes con ACC $(17,18)$. Por tal razón, puede dilucidarse la evolución clínica de este menor en el desarrollo de esta alteración y más aún los logros alcanzados en la trazabilidad del proceso de intervención y rehabilitación encontrado en sus primeros cuatro años de vida, por lo tanto, los hallazgos en el caso sugieren que a más temprana edad se logre, la detección del cuadro, los logros serán mucho más alentadores y de mejor pronóstico para el paciente. La multidisciplinariedad es efectiva en el proceso de intervención porque asegura el trabajo de todo un equipo de salud que trabaja mancomunadamente en bien del paciente y la familia véase Tabla 3 en lo relación al Seguimiento multidisciplinar en el caso clínico ACC.

Cabe destacar, la participación de todo un equipo de profesionales en salud, incluyendo un equipo Psicosocial conformado por psicología y trabajo social, realizaron una intervención basada en los parámetros de la técnica de modificación de conducta (cognitivo, comunicacional y emocional y psicoeducación) encontrando retraso mental global del desarrollo, además se reforzó proceso de aprendizaje, habilidades sociales y entrenamiento a los padres en el manejo de respuesta automática en tareas concretas y básicas. Así mismo, la terapia domiciliaria para seguimiento del entrenamiento logró cambios mentales de los padres, apropiándose del caso como coterapeutas y cuidadores del niño, mejorando habilidades sociales en el niño con respecto a su hermana y otros niños.

Es importante, resaltar la intervención del equipo psicosocial y las áreas no médicas, en lo que respecta la constancia y seguimiento oportuno a través de sus evaluaciones y estos procesos de intervención, lograron desarrollar estrategias y sugerencias para el resto de profesionales involucrados en el caso: pediatría, neurología, genetista, gastroenterología, oftalmología (19), odontología y nutrición (20).

La participación del genetista está justificada en el estudio en la medida en que es frecuente la asociación con otras malformaciones y con síndromes, aún en las formas aparentemente aisladas $(21,22)$, los estudios permitieron especificar el diagnostico (23).

A razón de lo anterior, se afirma que la intervención integral multidisciplinar en el tratamiento de la ACC es la más recomendable, ya que intermedia de manera efectiva en los aspectos biopsicosociales del 
paciente, promueve un estilo de vida saludable que previene la aparición de otros posibles trastornos del comportamiento, lo que permitió verificar que la ACC no estaba asociada a otra patologías, lo que permitió corroborar en otros estudios desarrollados por la Fundación Española de Enfermedades Huérfanas (24), que el tratamiento con otras áreas: fisioterapia, logoterapia, fármacos antiepilépticos y psicoterapia.

La ACC aislada parece estar relacionada con un pronóstico más favorable que la $\mathrm{ACC}$ asociada, ya que cuenta con hasta un $80 \%$ de casos con un pronóstico bueno. No obstante, debe informarse a los padres de que pueden presentar dificultades de aprendizaje (asociadas a lentitud, déficit de atención) que requieren rehabilitación apropiada. Tal como el caso presentado, donde el pronóstico se acentúa en el seguimiento oportuno con las respectivas formulaciones médicas y no médicas, que debe mantenerse dentro de la intervención y contexto familiar, específicamente los padres, acentuando una adecuada y oportuna estimulación en el niño, siguiendo un plan de trabajo $\mathrm{y}$ de actividades que propicien estos cambios en habilidades sociales, psico-educación enfocada a una adherencia optimizada en todos los procesos, desde la ingesta de medicación hasta la asistencia controles médicos especializados que determinan la evolución de la enfermedad.

En este sentido se comprende destacar que suele observarse el riesgo de esta malformación dependiendo de las comorbilidades que susciten tal es el caso de las visuales, retardo mental global del desarrollo, alteraciones a nivel nutricional, trastornos del comportamiento, deterioro cognitivo, entre otros. De allí, que teniendo en cuenta los hallazgos en el presente caso clínico se concluye que las estrategias adoptadas generaron cambios significativos en el contexto familiar y social que propenden una mejoría en el bienestar físico, social, y emocional del niño, garantizando una mejor calidad de vida en su entorno y evolución clínica del paciente.

\section{Correspondencia}

Francis Araque Barboza

Correo electrónico: faraque@unimetro.edu.com

\section{REFERENCIAS BIBLIOGRÁFICAS}

1. Schinzel A. Postaxial polydactyly, hallux duplication, absence of the corpus callosum, macrencephaly and severe mental retardation: i a new syndrome? Helvetica pediatric acta. 1979; 34 (2): 141-6.
2. Stoll C, Dott B, Roth MP. Associated anomalies in cases with agenesis of the corpus callosum. American Journal of Medical Genetics. 2019; 179 (10): 21012111. Doi: 10.1002/ajmg.a.61330

3. Ballardini E, Marino P, Maietti E, Astolfi G, Neville A. Prevalence and associated factors for agenesis of corpus callosum in Emilia Romagna (1981-2015). European Journal of Medical Genetics. 2018; 61(9): 524-530. Doi: 10.1016/j.ejmg.2018.06.004

4. Lagares A, Haro A, Crespo P, Ceballos V, Rodríguez R, Conejero J. Agenesia del cuerpo calloso. Discordancia clínico-radiológica. Análisis tras 15 años de experiencia. Rehabilitación. 2011; 45(3): 208216. (Fecha de acceso 15 de agosto del 2021). Disponible en: http://pesquisa.bvsalud.org/portal/ resource/pt/ibc-90011

5. Jiang Y, Qian YQ, Yang MM, et al. Whole-Exome Sequencing Revealed Mutations of MED12 and EFNB1 in Fetal Agenesis of the Corpus Callosum. Frontiers in Genetics. 2019; 10: 1201. Doi: 10.3389/ fgene.2019.01201

6. El Ameen N, Ibrahim M, Mounir S. Fiber Tractography and diffusion tensor imaging in children with agenesis and dysgenesis of corpus callosum: A clinico-radiological correlation. International Journal of Pediatrics. 2019; 7(8): 9903-9915. Doi: 10.22038/IJP.2019.39349.3354

7. Martínez C, Granados E, Sandoval C, Zepeda R, Elizalde I, Casian G. Agenesia de cuerpo calloso y otras disgenesias cerebrales. Evaluación, clínica y diagnostico radiológico. Anales de Radiología México 2004: 4:281-286. (Fecha de acceso 15 de agosto del 2021). Disponible en: https://www. medigraphic.com/pdfs/anaradmex/arm-2004/ arm044g.pdf

8. Rodríguez J, Martínez J, Renté Y. Agenesia parcial del cuerpo calloso en un infante. MEDISAN. 2016; 20(8): 0-0. (Citado el 28 de octubre del 2020). Disponible en: http://scielo.sld.cu/scielo.php?script= sci_arttext\&pid=S1029-30192016000800013

9. Bartolomé E, Cottura J, Britos R, Domínguez R. Colpocefalia y agenesia parcial de cuerpo calloso asintomáticos. Neurología; 2016 31(1): 68-70. http://www.elsevier.es/es-revista-neurologia295-articulo-colpocefalia-agenesia-parcial-cuerpocalloso-S0213485313002843

10. Margari L, Palumbi R, Campa M Operto F, Buttiglione $\mathrm{M}$, Craig F, et al. Clinical manifestations in children and adolescents with corpus callosum abnormalities. J. Neurol. 2016; 263:1939-1945. Doi: 10.1007/ s00415-016-8225-x Fisher, R. S., Cross, H. J., French, J. A., Higurashi, N., Hirsch, E., Jansen, F. E., \&

11. Aljure-Reales V, Rangel-Carrillo JJ, Ramos-Garavito JD, Rodríguez JA, Rodríguez JS. Agenesia del cuerpo calloso: un tema poco conocido. CES Med 2017; 31(2): 172-179. (Fecha de acceso 15 de agosto 
del 2021). Disponible en: http://www.scielo.org.co/ pdf/cesm/v31n2/0120-8705-cesm-31-02-00172.pdf

12. Scheffer I. Clasificación operacional de los tipos de crisis por la Liga Internacional contra la Epilepsia: Documento-Posición de la Comisión para Clasificación y Terminología de la ILAE. Epilepsia. 2017; 58(4): 522-530.

13. Folliot-LeL, ChadieA, Brasseur-Daudruy M, Verspyck E, Saugier-Veber P, Marret S. Neurodevelopmental outcome in prenatally diagnosed isolated agenesis of the corpus callosum. Early Hum Dev. 2018; 116:916. Doi: 10.1016/j.earlhumdev.2017.10.004

14. Brown W, Lynn P. The neuropsychological syndrome of agenesis of the corpus callosum. Journal of the International Neuropsychological Society. 2019; 25(3): 324-330. Doi: 10.1017/S135561771800111X

15. Chokkian J. Agenesis of corpus callosum. Indian Journal of Continuing Nursing Education. 2019; 20(2): 97-101. DOI: 10.4103/IJCN.IJCN_13_20

16. Suárez-Villalobos P, Peña JA, Calzadilla L, Ruíz M, Cruz ML. Síndrome de Aicardi: Descripción de cuatro casos venezolanos. Investigación Clínica. 2010; 51(3): 415-422.

17. Urrestarazu E, Murie M, Viteri C. Manejo de la primera crisis epiléptica y del status en urgencias. Anales del Sistema Sanitario de Navarra 2008; 31: 61-73.

18. Antonio-Luna E, Bayliss-Amaya L, Guerrero-Juárez V, Estrada-Galindo Á, Ortiz-Maldonado J, RomeroCano D, et al. Síndrome de Aicardi. Archivos de Neurociencias. 2014; 19(4): 215-220.
19. Promelle V, Demeer B,. Milazzo S. Patologías congénitas en oftalmología."EMC-Pediatría. 2020; 55(2): 1-13.

20. Henderson G, Beerse M, Liang H, Ferreira D, Wu J. Improvement in overground walking after treadmillbased gait training in a child with agenesis of the corpus callosum. Physical Therapy. 2020;100(1): 157-167. Doi: 10.1093/ptj/pzz144

21. Rodríguez J E, Martínez JA, Renté Y. Agenesia parcial del cuerpo calloso en una infante. Medisan. 2016;20(8): 2001-2006.

22. Rocha E, Masotto B, Rittler M. Agenesia de cuerpo calloso en recién nacidos. Rev Hosp Mat Inf Ramón Sardá. 2017. 2(2):0-0.

23. Govil-Dalela T, Kumar A, Agarwal R, Chugani HT. Agenesis of the Corpus Callosum and Aicardi Syndrome: ANeuroimaging and Clinical Comparison. Pediatr Neurol. 2017; 68:44-48. doi: 10.1016/j. pediatrneurol.2016.12.002

24. Fundación Española de Enfermedades Huérfana. Detalles de la patología: Agenesia del Cuerpo Calloso.Madrid:FundaciónEspañoladeEnfermedades Huérfanas. (Citado el 28 de octubre del 2020). Disponible en: https://www.enfermedades-raras.org/ index.php $/$ component $/$ content $/$ article? $\mathrm{id}=3100$ \&idpat $=15$

Recibido: 19/03/2021

Aceptado: 08/07/2021 